\title{
THE PRODUCTION OF COMPLEX THIN FILM CIRCUITS
}

\author{
V. NEEDHAM \\ Lucas Aerospace Limited, Engine Electronics Division, Birmingham, England
}

(Received December 13, 1976)

\begin{abstract}
In-house manufactured thin film circuits are used by Lucas Aerospace in gas turbine engine fuel control systems where stability and reliability in a harsh environment are required.

The construction of a complex active thin film circuit is reviewed with emphasis on some less obvious aspects of production. Where component attachment and engineering techniques used are thought to be of special interest a more detailed explanation is given.

As is normal in the avionics industry a high degree of system integrity is required so that quality control is essential. Therefore, the role of Quality Engineering in the production plan is described.
\end{abstract}

\section{ACTIVE THIN FILM CIRCUIT}

A typical active thin film circuit is shown in Figure 1 together with the associated assembly drawing Figure 2. The thin film circuitry is based on nichrome resistors and gold conductors. Add-on components are in the form of beam lead devices (diode, transistors and integrated circuits) plus monolithic chip capacitors. All interconnection wires are gold and are attached by thermocompression ball and wedge bonding - gold to gold bonding metallurgy is used throughout the circuit build.

This circuit contains 36 resistors (the black strips in Figure 2) ranging in value from 50 to $150 \mathrm{kohms,}$ 12 beam lead devices and 5 capacitors plus interconnection wires.

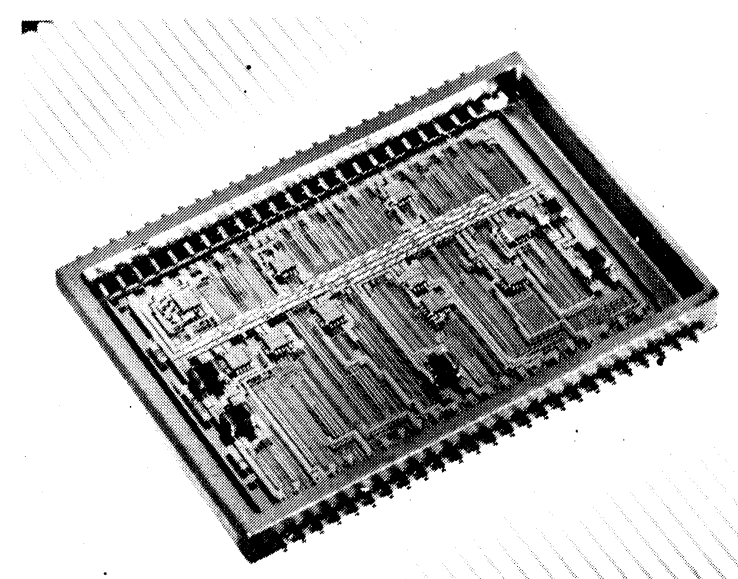

FIGURE 1 Active thin film circuit -8 tangent function generator. (Substrate size $3 \times 2 \mathrm{cms}$ )

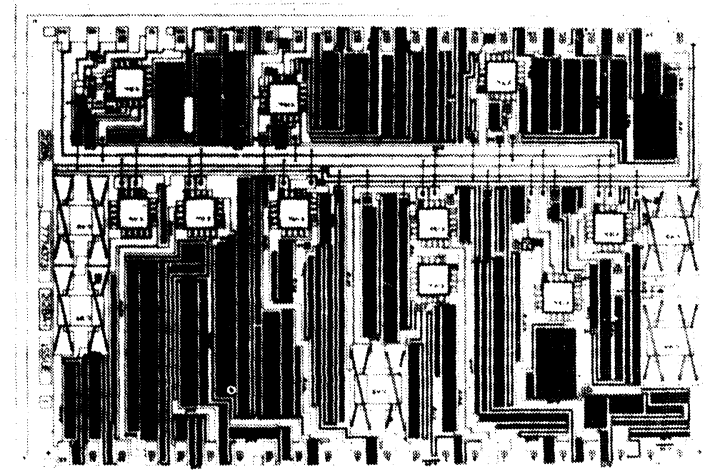

FIGURE 2 Circuit assembly drawing for active thin film circuit shown in Figure 1.

All thin film processing, assembling and testing is carried out in a class 100 , laminar flow clean room (Figure 3).

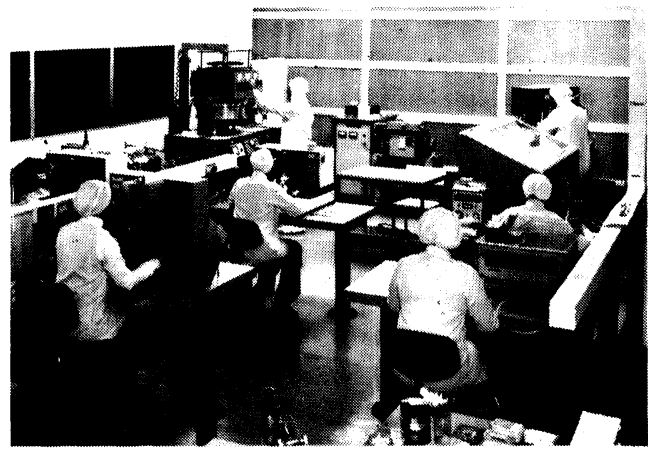

FIGURE 3 Thin film processing area. 


\section{THIN FILM PROCESSING}

Thin film deposition (gold and nichrome) is by R.F. diode sputtering under controlled temperature conditions so that subsequent stabilisation baking is not necessary. The prime requirement of the film deposition process is repeatability of thin film characteristics. Initially experience was obtained using nichrome sublimation and gold evaporation. These techniques proved difficult to control in production and some eight years ago the deposition techniques for both nichrome and gold were replaced by R.F. diode sputtering. Inter batch and batch to batch repeatability, using sputtering, is dependent upon accurate control of four parameters: bias voltage, pressure, temperature, time. An in-house equipment was built having closed loop control to monitor these parameters. Another essential production feature of the equipment is automatic programme control so that the operator's activity is restricted to loading and unloading the equipment.

Film to substrate adhesion is also of great importance and has been found to be dramatically enhanced by sputter etching the substrate, removing some $100 \AA$, after an initial chemical clean, prior to film deposition.

Circuit definition is achieved by a conventional 2 stage photolithographic process. The first stage is to define the entire circuit array in nichrome and gold followed by removing gold in selected areas to form resistors. Wet chemical etching for the first stage presents quite severe process constraints as a result of which nichrome undercut can rea dily occur. The problem of undercut is such that batch etching cannot be successfully employed and even with individual etching, the etch time margin between the removal of unwanted nichrome and the onset of undercut is small i.e. about one second. For fine line width resistor tracks (less than 5 thou" width) sputter etching of nichrome and gold is now used. Undercut effects by sputter etching are virtually non existent so that 'blanket' etch times can be used. Figure 4 shows the undercut effect due to wet chemical etching as opposed to the results obtained by sputter etching.

Compared with the cost of circuit assembly and testing the cost of a fully etched substrate is relatively low. To avoid assembling and testing substandard thin film circuits, each etched-out thin film is subjected to rigorous inspection relating to resistor tolerance and visual standards.

Using a customised multi-probe bed the value of each resistor and the ratio between specified resistors
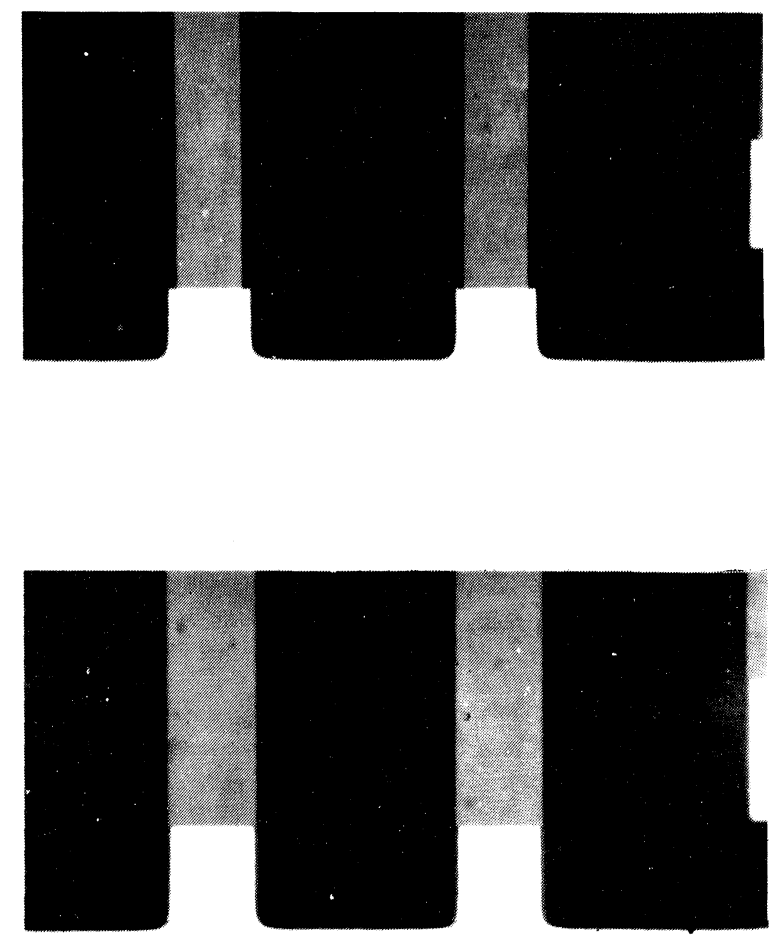

FIGURE 4 Etching of resistors (resistors are shown in black and are 5 thou" wide). (a) Undercutting effect due to wet chemical etching, (b) Zero undercutting with sputtered etching.

and the isolation resistance between non-connected parts of the circuit are automatically measured. The measuring equipment had a paper print out and is programmed to display rejection when measured values fall outside prescribed limits. Circuits which fail to meet the required measurement limits are rejected at this stage.

When evaluating new circuit types the measured values from several circuits are subjected to computer analysis, the results of which are displayed in the form of a histogram (Figure 5) from which repetitive errors are readily detected. When repetitive errors are indicated then an investigation is carried out. Firstly the photomasks are critically examined for damage and then specific resistor defining tracks are accurately measured and compared with the design specification. Discrepancy between measured photomask tracks and design requirements is corrected by modifying the cut and strip artwork and remaking the photomasks. 


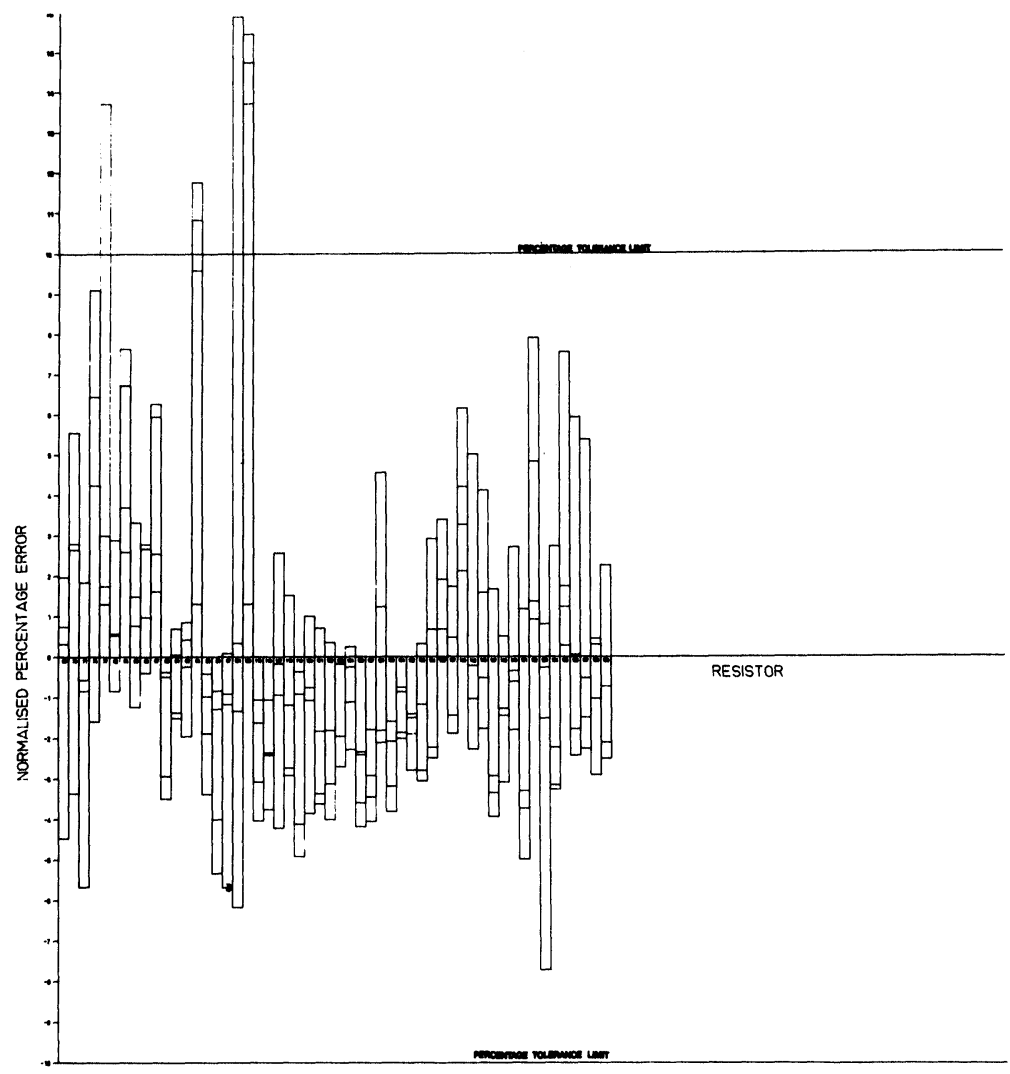

FIGURE 5 Normalised percentage error histogram for resistors.

Etched circuits which pass all value measurements are visually inspected for track damage (holes, scratches, etc) contamination and residual nichrome or gold. On the basis that nothing is ever perfect and that intense examination will always show some imperfection it is vitally important that the inspection standards employed are realistic if reasonable yields are to be obtained. Our experience suggests that continuous dialogue between Production and Quality is necessary to revise inspection criteria, if practical standars are to be established.

\section{CIRCUIT ASSEMBLY}

It should also be mentioned that each substrate is back metallised (by sputtering techniques) with nichrome, nickel and gold. This enables the thin film substrate to be solder bonded to the package base. Apart from this one operation all other joining techniques, as previously stated, maintain a gold to gold metallurgy.
The successful assembly of complex thin film circuits is dependent upon the following factors:

1) Bonding parameters

2) Equipment maintenance and setting

3) Defined bonding standards

4) Design criteria and presentation of instructions to operators

5) Rework capability

Justification for the selection of particular device types and forms, and assessment of the electrical performance of the devices are outside the scope of this paper.

In Lucas Aerospace bonding parameters (temperatures, pressures, etc) are derived by Circuitry Joining Engineers and written into process control documents which are used by production unless experience gained shows them to need modification.

Effective equipment maintenance is an obvious requirement as it is of critical importance to efficient machine operation and bonding. The key to success 
in this area is to differentiate clearly between maintenance and setting as, in general, entirely different skills are required. Usually, in the U.K., maintenance engineers (works engineers) have responsibility for keeping the equipment and services running and do not, as a rule, have the skills or appreciation of bonding requirements to undertake the fine setting of equipment. Setters, on the other hand, have full appreciation of the bonding standards so that they can assess bonding faults and take corrective action. It is therefore essential that the responsibilities of maintenance engineers and setters are clearly defined. It is somewhat more difficult to teach operators how to realise when setting changes are required. Ideally, each machine operator should be his own setter although, unless extreme care is taken, each would develop his own personal machine setting standards. The precise division between maintenance and setting will depend on company policy, pay scales and any other local conditions applying to any particular manufacturing unit.

Defining bonding standards is an obvious requirement, relating to such items as the degree of deformation, the position of welds on wires or on a device leg, and the positioning of wires in relation to the bonding pad area etc. A general failing of most bonding equipment is the inability to be able to have high magnification with a good field of view. This latter requirement is essential when assembling circuits of the visual complexity shown in Figure 1, as operators need to be able to orientate themselves with respect to the surrounding circuitry. On the other hand, proportionately higher magnification is required for both accurate component positioning and for assessment of the work standard. Operators naturally tend to work at low magnification at the expense of weld quality. However, inspectors are not confronted with the same constraints and work at high magnification and identify faults which go undetected by the operators. The author's impression is that a lot of the problem lies with the equipment manufacturers who do not realise the importance of the type of optical systems used on their equipment. It would seem that much expertise is related to the mechanics of the bonding equipment but that the optics are given secondary consideration. This leads not to the use of poor quality optics but to the employment of unsuitable optical systems.

Attention to circuit assembly design criteria is important and the Production Department has to be fully involved during the design of the circuits to ensure that their requirements are appreciated and incorporated. Circuit engineers otherwise may tend to consider the circuit design purely from the aspect of circuit function and packing density resulting in a circuit that cannot be manufactured to an acceptable yield. Most design rules are obvious in retrospect, but often forgotten during design unless clearly defined. For example, devices should not be positioned so that the bonding tool fouls other components or that rectification cannot be carried out without having to move surrounding components.

Each manufacturer has his own sets of design constraints, and the task of the Production Department at the circuit design stage is to ensure that its needs are incorporated in the rules and are implemented in practice.

A predominant cause of rejection on inspection is assembly errors, i.e. devices wrongly oriented, link wires going to the wrong places etc. Thus the presentation of assembly information to the operator is of paramount importance. For operator use, the circuit drawing assembly (Figure 2) has two major faults:

a) Lines appear on the drawing which are not present on the etched circuit. This is peculiar to the way in which the tape controlled flat bed plotter, on which the drawings are produced, is programmed. These extraneous lines cause confusion.

b) Too much information is present on one drawing.

The solution to these two problems is:

a) Photograph an etched thin film circuit and from this produce a master circuit pictorial representation.

b) Derive copies of the master representation and on each display only one type of device or operation. For instance, one copy may show only the positioning and orientation of beam lead diodes, whilst another shows the position and orientation of one type of beam lead integrated circuit. By this means a circuit set of drawings is derived, each defining only one aspect of the final assembly.

Having reduced the main assembly drawing to a set of sub-assembly drawings, other problems arise, because the majority of the assembly operation requires the use of a microscope. The use of a drawing in conjunction with a microscope is problematic for two reasons:

a) the sub-assembly drawing is at a different scale from the image of the circuit presented through the microscope. 
b) The transcription of information from drawing to circuit involves a head movement and change in eye focus which causes fatigue and reduced retention of information.

Ideally all the assembly information should be shown on the circuit being assembled, as practised in printed circuit board assembly. However, for the size and complexity of circuits being considered this is not practicable.

A partial solution is to include marks on the circuits which allow the operator to connect up various sections correctly. Examples are shown in Figure 6. A mark on one leg of a beam lead device (A on Figure 6a) is oriented by an indentation of the reference pad in the circuit (A on Figure 6b).

Similarly, ball and wedge link wire extremities are
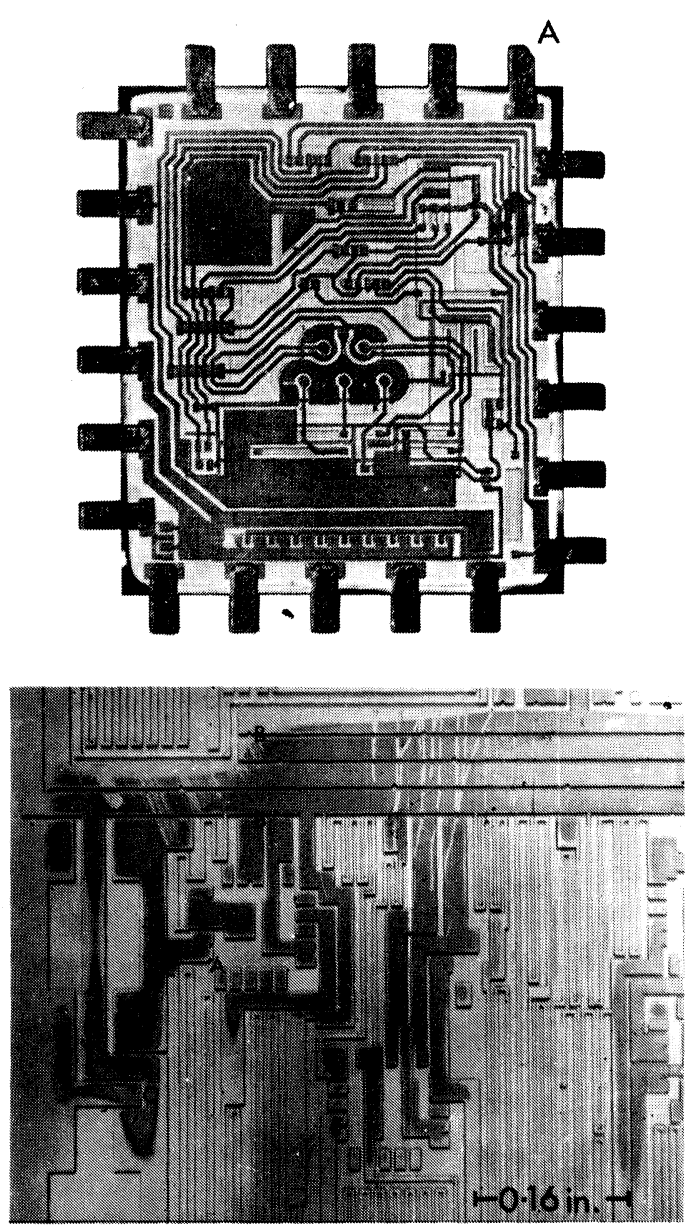

FIGURE 6 Assembly aids on circuits. (a) Beam lead device showing oreintation indentation. (b) Thin Film Circuit with assembling aids. denoted by notches (B, B on Figure $6 \mathrm{~b}$ ) in the conductor lines. Matching notches are horizontal or vertical to each other; link wires running diagonally are discouraged. Rectangular notches in conductor tracks are used to denote the extremities of conductor track stitching (one end of such a stitching position is shown at $\mathrm{C}$ in Figure $6 \mathrm{~b}$ - the magnification of Figure $6 \mathrm{~b}$ is too high to show the other end). This last technique is used to improve conductor track conductivity in critical areas. The increased design and artwork preparation time incurred by adding these visual aids to assembly are more than justified by the reduction in operator assembly errors.

Beam lead device bonding is accomplished using Kulicke and Soffa Model 576 bonding machines with Tempress Tools. Typical bonding faults (apart from positioning, which is operator dependent) range from insufficient beam deformation and insufficient device 'bugging' to isolated beams not being bonded at all. Usually all these faults can be traced to incorrect machine setting and tool maintenance. Remedial action can be taken to rectify instances of insufficient deformation and beams not welded, by using a 'nail' bonder which can be operated on individual beams. Repair can also be accomplished by shaving the beams on a device, thereby removing a particular device from a circuit - a replacement device being added in the normal manner, subject to inspection approval to do so. Compared with alternative device attachment techniques, beam lead bonding is elegant and eminently suited to thin film circuitry.

When device types are required which are not available in beam lead form an alternative device mounting technique is used which incorporates the use of a ceramic carrier (Figure 7). The silicon device is eutectically bonded to suitably metallised alumina carrier and electrically connected to the carrier terminals. These devices are received mounted on a thick film printed test card in a 'burnt-in' state. The connections between carrier and test card are made using gold tapes which are parallel gap welded to the carrier and the test card. The carrier mounted device is removed from the test card severing the gold tape adjacent to the weld on the thick film conductors, transferring the carrier to the thin film circuit and parallel gap welding the free ends of the gold tape onto the gold thin film bonding areas. Orientation and position of carrier mounted devices on the circuit are self evident and there is no need for special indication.

The method of attaching chip capacitors to the circuits is as follows. Gold tapes are parallel gap welded to the top and bottom of the component and 

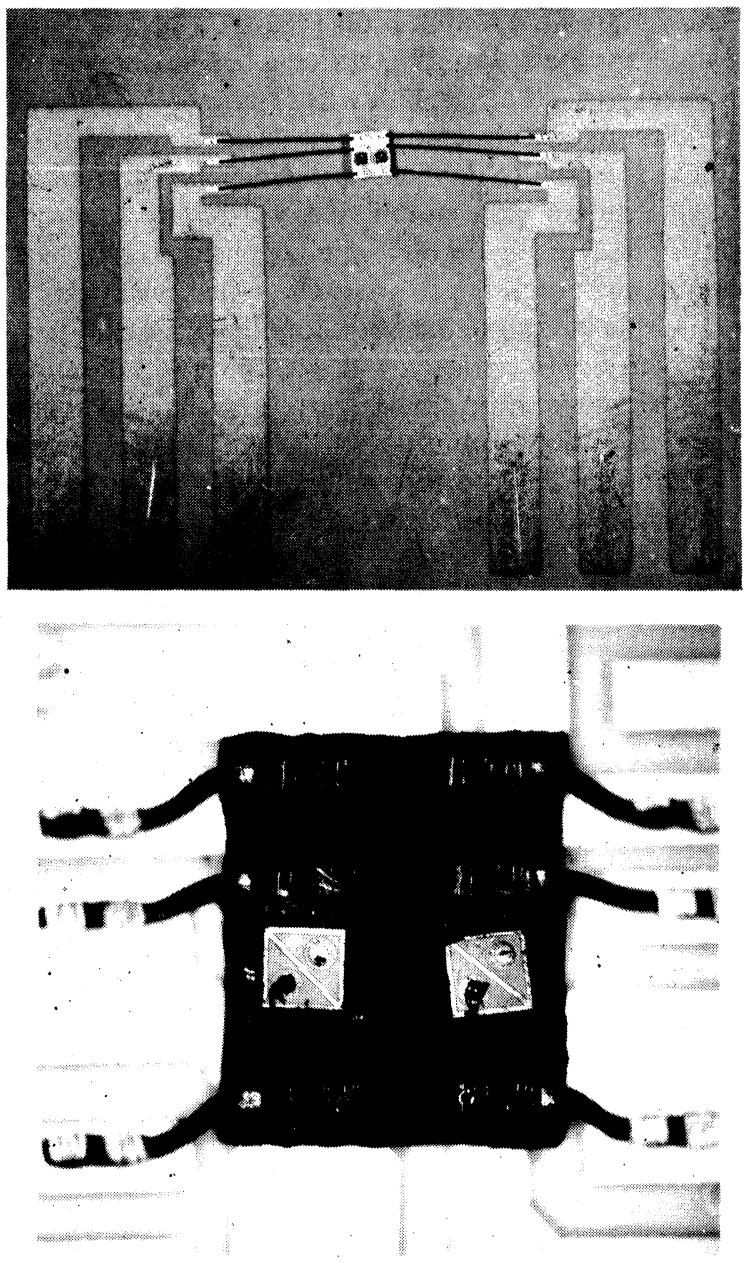

FIGURE 7 Ceramic carrier mounting technique for semiconductor devices (carrier $1 \frac{1}{2} \mathrm{~mm}$ square). (a) Low magnification. (b) High magnification.

to the thin film to give both electrical and mechanical connection to the circuit. Cross-over wires and connection from the thin film circuit to the internal package lead-out terminations are made with fold wire using thermo-compression ball and wedge bonding.

\section{FUNCTIONAL TRIMMING AND TESTING}

Active functional trimming of the thin film circuits is performed on a computer controlled laser/A.T.E. system (Figure 8). All trim and test instructions are held on magnetic tape. Circuits to be adjusted are held in a special jig which is multiplexed to the test equipment via a specialising card. The system is organised so that correlation between the thin film

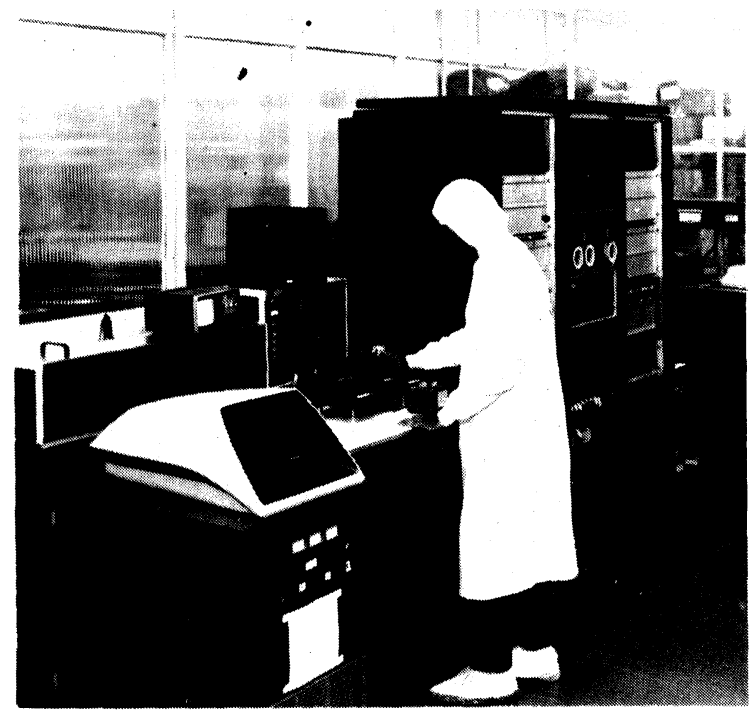

FIGURE 8 Computer controlled laser trim and automatic test equipment.

circuit, specialising card and test schedule is required before the system can operate. Because of the laser spot size used $(0.8 \mathrm{mil})$, it is important that the plane of the thin film circuit surface and the focal plane of the laser are made co-incident if consistent out track widths are to be maintained over the circuit. This is accomplished by having the circuit mounted on a hemi-spherical ball and socket which can be raised to bring the substrate surface into contact with three pins, the tips of which are mechanically fixed in the focal plane of the laser. Following positioning of the circuit in the jig the operator will then eliminate theta error between the co-ordinates of the thin film circuit and the laser head movement and zero the system. Using the closed circuit television, (which focuses through the laser head), theta error between the circuit ordinates and the C.C.T. graticule can be nullified by making a small rotational adjustment to the circuit retaining jig. After this adjustment is completed the laser homes to the vicinity of a reference on the circuit. Using manual control the centre of the graticule and the circuit reference point can be made co-incident, after which the laser head $\mathrm{X}, \mathrm{Y}$ counters are effectively zeroed. From this stage on, the computer is fully in command and apart from unloading, the operator's task is complete. After lidding, (seam welding in an inert atmosphere), the circuit is re-tested using the test part only of the laser/A.T.E. system. A second station on the system enables circuits to be tested at operational temperatures. 


\section{QUALITY CONTROL}

The many responsibilities of the Quality Department for product assurance embrace all aspects of circuit manufacture, from circuit design to acceptance testing. However, only the process control responsibility will be considered here.

All production processes are fully documented and the quality requirements at each stage are detailed to ensure that production and inspection personnel understand the standards required.

Two prime requirements of a standard are:

a) that they reflect contractual requirements and establish codes of practice.

b) that they are practicable and economical to apply.

Establishing a balance between these two factors can be difficult, as initial standards are often unnecessarily severe resulting in high levels of product rework and scrap. An example of this situation arose when beam lead devices were first used in Lucas circuits. Gne of the bonding standards called for rejection of any device which, after bonding, had one or more of its beanis overhanging the thin film pad to which it was bonded. For a variety of reasons this standard was difficult to achieve. The high levels of circuit rework and scrap caused by adherence to the standard regarding 'overhang' were unacceptable and a request was made by production for a relaxation of the standard. On the basis of experimental evidence the standard was revised to allow 'overhang' of up to $30 \%$ of the device beam width. The adoption of this revised standard had an immediate and beneficial effect in production. However, this revised standard introduced a side effect, i.e. that of determining 30\% of a beam without resorting to lengthy precision measurements.

Subjective requirements are perhaps, more problematic than most and can easily cause production problems. Again an example is sited which illustrates this point. At all stages of visual inspection of thin film circuits there is an in-house requirement that the circuit be free from contamination. On the basis that nothing is ever free from contamination of one form or another, this requirement is virtually impossible to implement, as what is regarded as being clean by one person is unacceptable to another. This dilemma is very difficult to resolve indeed and as yet, no ideal solution to it has been found. Tests on effects of known contaminants intentionally applied to thin film circuits have led to additions to or modifications of certain processes. On the basis of the results of these actions the standard has been re-defined in such a way that, although the subjective content of the inspection has not been eliminated, the production situation has been greatly eased.

Apart from process inspection stages necessary to ensure that mandatory criteria are met, the Quality Department provides information feedback to production in the form of reject and scrap analysis etc. To be effective in production control, information feedback has to be rapid. Too often, information relating to current production problems is purely of historical interest only by the time it is available. A system is now in operation which overcomes the information feedback time delay. This systems P.I.A.S., (Production and Inspection Analysis System) is computer controlled and provides a daily updated analysis of process yields, rework and analysis. Although originally conceived for process quality control purposes the scope of the system has been expanded to cover the requirements of production control. Input data is derived on special pass or rejection cards in the production area. Production pass cards record the individual circuit identification and stage number together with the lot number (if applicable) of components added for each circuit passing through a particular work station. Conversely, rejection cards identify the work concerned, the production stage at which the work failed and a serialised identification of the faults found. From the information, the built record of every circuit can be derived (a mandatory requirement). Also the system can be interrogated. For instance, particular device type failure can be correlated to circuit types, application batch date numbers, etc.

From the aspect of production control the P.I.A.S. daily print out shows the status of all work in the production shop and the loading on all work stations. P.I.A.S. has proved to be a very powerful tool for both Production and Quality Departments.

\section{CONCLUSION}

Using a description of a 'Lucas' thin film circuit as a framework for discussion some, less commonly reported, aspects of circuit production have been presented.

Although technologies and procedures differ between manufacturers some generalised conclusions can be made:

a) Manufacturing procedures and inspection standards are as essential to production as the technologies used. 
b) Standards and procedures need to be reviewed constantly and revised where appropriate.

c) When manufacturing visually complex circuits the presentation of assembly and inspection information requires careful consideration.

\section{ACKNOWLEDGEMENTS}

The author is indebted to the Directors of Lucas Industries and Lucas Aerospace Limited for their permission to publish this paper.

The author would also like to thank his colleagues in many departments, on whose work this paper is based.
The original of this paper was given at the 1976 International Microelectronics Symposium held in Vancouver October 11-13, 1976. The author is grateful to ISHM/USA for permission to publish this paper.

\section{REFERENCE}

1. W. C. Dolman, "The use of a low-cost, medium-sized computer installation in a hybrid design and manufacturing facility". Proc. 1976 International Microelectronics Symp. (Published by ISHM/USA, pp. 7-14, 1976. 

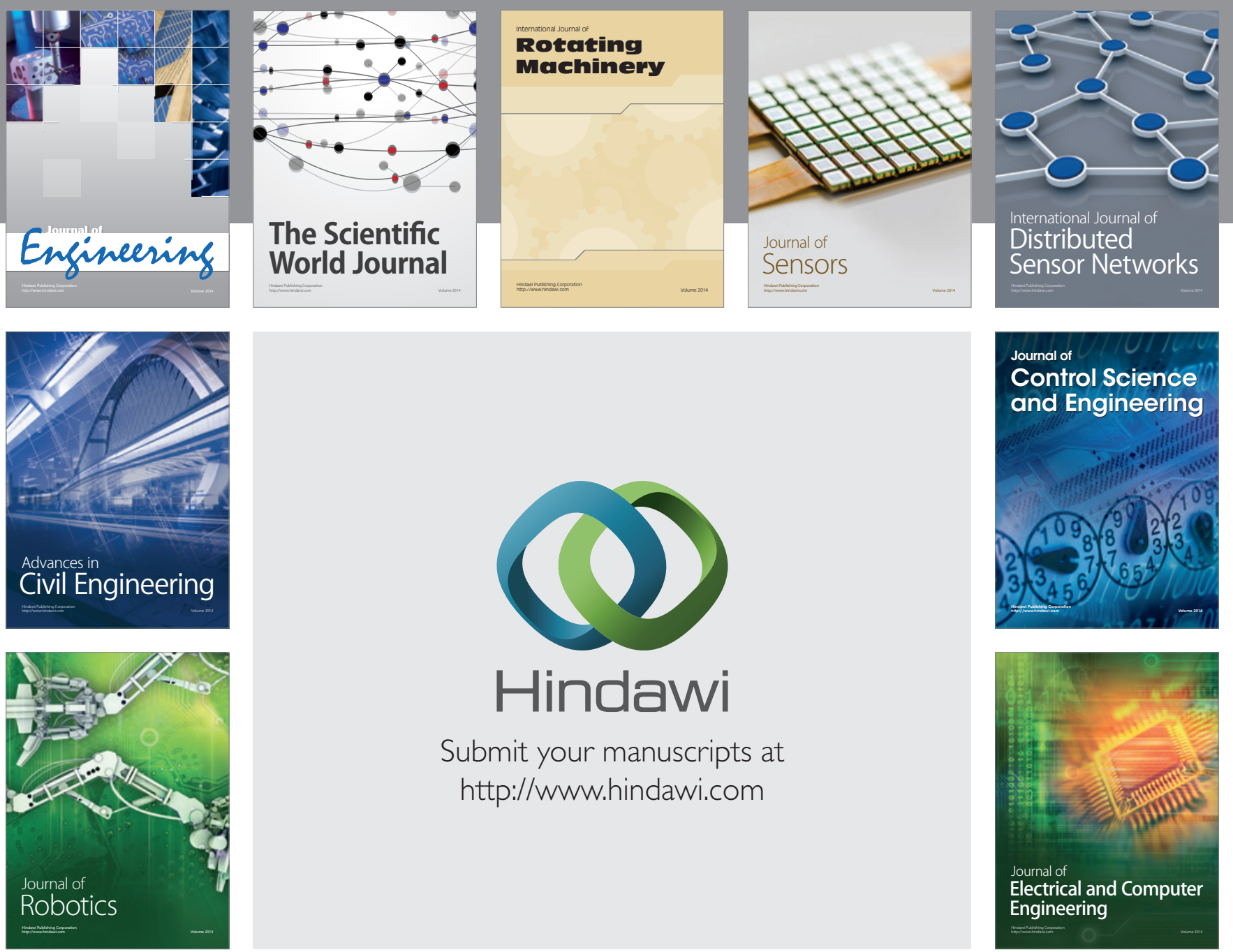

Submit your manuscripts at

http://www.hindawi.com
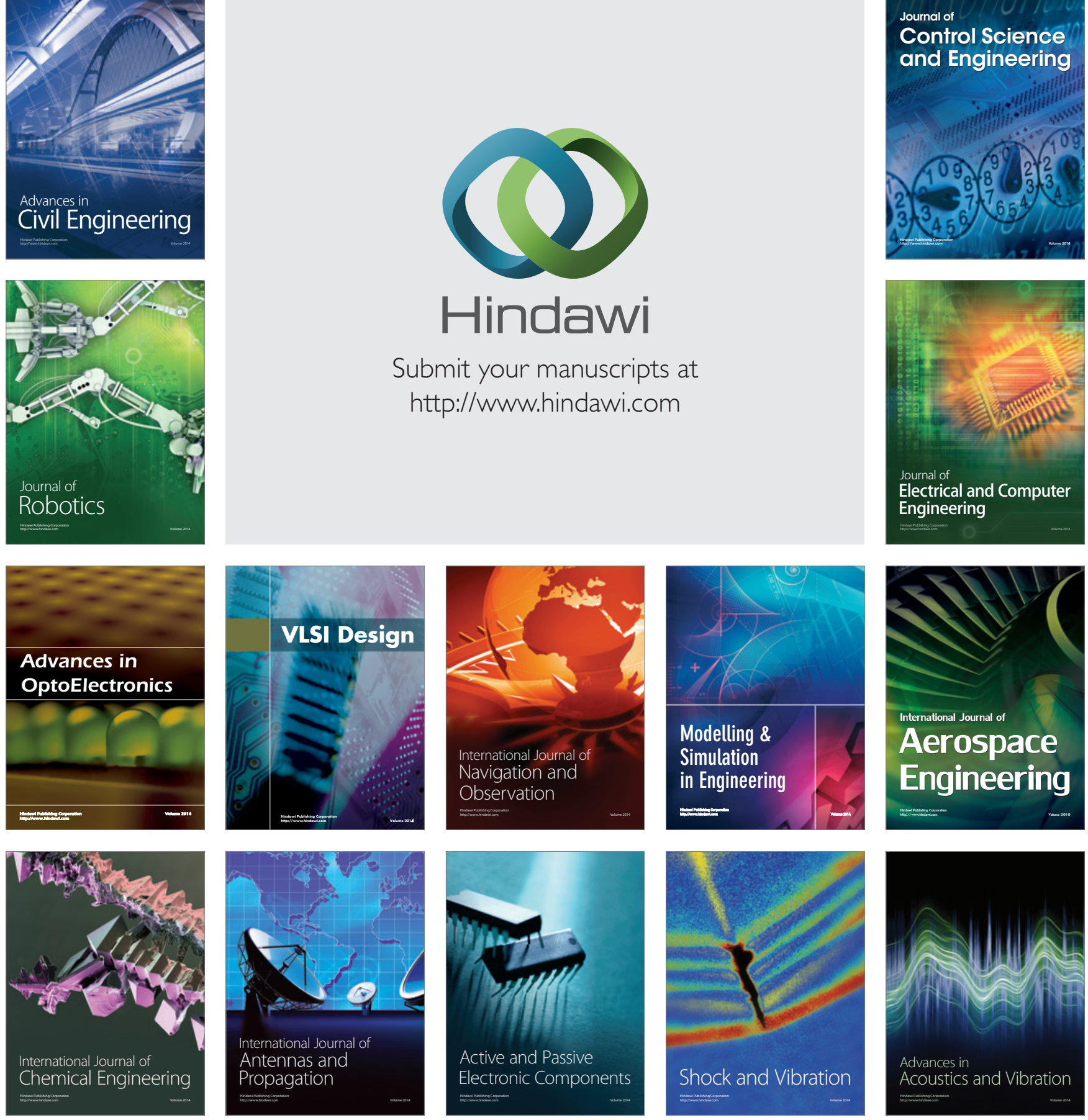